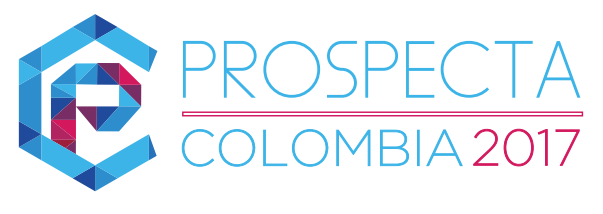

\title{
Canvas adaptado para los emprendimientos del posconflicto
}

Canvas adapted to post-conflict ventures

Juan Carlos Díaz Dávila*

* Candidato a Magister en Administración de Empresas. Especialista en Gerencia de Negocios Internacionales y Profesional en Administración de Empresas. Experiencia de varios años en empresas y entidades de los sectores público, privado y del social en dirección, gerencia, consultoría, asesoría, docencia y capacitación en áreas económico-administrativas. Correo electrónico: juank2607@gmail.com 


\section{Introduccion}

Después de casi cinco décadas de violencia generalizada en el país, con actores que apelaban a todo tipo de justificaciones para lograr sus objetivos, las cuales se podían considerar como las causas del conflicto; aparecen múltiples temas de investigación en diversas disciplinas en Colombia y en el exterior. En el siguiente texto, Vargas logra sintetizar gran parte de ellas y brinda un panorama global de lo que el conflicto representa:

En la Colombia contemporánea la violencia se presenta en muchos ámbitos de la vida nacional y hay un conflicto interno armado que tiene ya casi cuatro décadas de duración, en proceso creciente de profundización. En esto inciden la presencia de actores que apelan a la utilización de la misma como recurso para solucionar conflictos o conseguir objetivos de diverso tipo y factores de orden estructural que hunden sus raíces en la configuración histórica del país, que han dado como resultado estructuras socioeconómicas y políticas excluyentes que impiden el ejercicio de la ciudadanía para una buena parte de la población, los cuales diferenciamos de otros facto res posteriores que han contribuido a su reproducción y de los factores específicos que se encuentran en la base del surgimiento de las organizaciones guerrilleras. Dentro de los [actores de orden estructural se encuentran, además de la persistente tendencia histórica a utilizar la violencia para obtener objetivos políticos, las estructuras de exclusión o inclusión "perversa", socioeconómicas, políticas y regionales. junto con una cultura política autoritaria refractaria a los comportamientos democráticos, todos los cuales forman una especie de telón de fondo.

Dentro de los segundos, aparecen otros factores que van a ayudar a la reproducción de la confrontación, el narcotráfico en primer lugar, y especialmente los cultivos de uso ilícito, que se vuelven fuentes de rentas para la financiación de la guerra; el colapso del aparato de justicia como elemento de regulación de las conductas sociales y la disparada de la impunidad, la perdida de la confianza como valor social de cohesión, conductas delincuenciales y corruptas asociadas a la gestión del Estado (2006, p. 171). 
Con la llegada de Santos a la presidencia de Colombia -durante dos periodosse inicia un proceso de negociaciones en el cual se concentran las esperanzas de un porvenir mejor para las nuevas generaciones y la reincorporación de millones de Colombianos a sus vidas antes de la guerra, y de otros cuantos miles de combatientes a la vida civil, en donde su principal misión será la de ser agentes económicamente productivos y políticamente activos; No obstante lo anterior, los tiempos de posconflicto en otros países dejan mucho que desear en el cumplimiento de esas metas de bienestar, como lo describen detalladamente las conclusiones del estudio de Garzón (2003) - que se presenta a continuacióndonde evidencia un posible panorama de lo que en realidad puede ocurrir, desde la perspectiva comparada con otros Estados y sus situaciones de posconflicto.

Bajo el presupuesto de que la guerra es la fuente primera de las muertes, la firma de los acuerdos va acompañada de la expectativa de que finalizada la confrontación, la tranquilidad reinará. Sin embargo, el nuevo escenario, antes que presentar una baja en los niveles de violencia, los aumenta e incluso los estimula, de modo tal que supera de manera sorprendente el panorama que lo antecedía. Lo que sucede entonces es que, si bien la violencia política, de hecho, baja notablemente, la violencia común se generaliza y aumenta. Esto se puede observar de manera explícita en los casos del Salvador y Guatemala.

[...] se ha llegado a pensar que un escenario postconflicto generará, por un lado, el esclarecimiento de los crímenes llevados a cabo en medio de la confrontación armada, acompañado por el enjuiciamiento de los victimarios, y por el otro, el establecimiento de la justicia como función primordial del Estado. A pesar de estos supuestos, la terminación de la guerra pocas veces trae consigo el enjuiciamiento de miembros de los grupos armados y la construcción de una eficiente maquinaria judicial. En los casos de El Salvador y Guatemala, tanto las Fuerzas Militares estatales como los grupos paramilitares y las organizaciones insurgentes, llevaron a cabo acciones violatorias de los derechos humanos e infracciones al derecho internacional humanitario, las cuales la mayoría de las veces, quedaron impunes en medio de la terminación de las guerras por la vía negociada. 
[...] es que es difícil construir una relación segura entre un escenario de postconflicto y un mayor desarrollo económico. En el caso del Salvador, por ejemplo, las cifras de la CEPAL demostraron que mientas el crecimiento del PIB durante la década de los ochenta —en plena guerra-, registró un promedio de 0.4 \% decreciente, durante los años noventa - luego de firmados los acuerdos de paz- repuntó a un 4.4 \%. En ese momento algunos académicos y economistas presentaron estos indicadores como la muestra de que la paz afectaba positivamente la economía. Sin embargo, la desaceleración del PIB en 1996 - año en el que bajó al 1.8 \% - y las alarmantes estadísticas —a fines de 2000, el 80 \% de las familias no alcanzaba a cubrir el costo de la canasta ampliada de mercado, un 23 \% vivía en condiciones de miseria, y alrededor del $70 \%$ de la población económicamente activa estaba en la informalidad - terminaron por poner de manifiesto que el rumbo de la economía estuvo lejos de determinarse en la mesa de negociación, a pesar de todas sus buenas intenciones. (pp. 126-130).

Según lo anterior, se debe considerar el elevado nivel de incertidumbre que se plantea en el macro ambiente del posconflicto, teniendo como imperativo para la sociedad el logro de las mejores condiciones de vida posibles para el ser humano en el entorno en el que se desarrolla su vida. Éste ha sido un asunto que ha sucitado debates permanentes al mas alto nivel tanto académico como de las entidades de regulación gubernamental y varias ONG, relacionados con los aspectos ontológicos y epistemológicos del mismo, asunto que puede verse reflejado en el siguiente texto que ilustra claramente dicho debate:

Es necesario insistir en una mejor distinción entre crecimiento y desarrollo, destacando que del crecimiento podría decirse que es económico, pero del desarrollo que es humano. Entendido el primero como la creciente disponibilidad de medios para satisfacer las necesidades materiales, sociales y espirituales y el segundo como su utilización para los fines propios de unas condiciones dignas de vida o de bien-estar o mejor-vivir de las personas (Colmenares, 2007, p. 1). 
Teniendo en cuenta que el sistema capitalista ajustado a las leyes del mercado, tiene como uno de sus axiomas fundamentales que el anhelado progreso se dará sólo en la medida en que las empresas con su crecimiento generen las ganancias suficientes para alimentar el ciclo de: inversión-beneficios-reinversión (Mora, 2012); es necesario priorizar estrategias que propicien la prosperidad de la nación mediante el apoyo al emprendimiento para la generación de nuevas empresas y nuevas unidades de negocio de las ya existentes. Esto se ha venido consolidando en los últimos años en Colombia gracias a las leyes de fomento que buscan impulsar la dinámica empresarial con recursos canalizados a través de fondos, como es el caso del Fondo Emprender, que beneficia especialmente a los emprendedores con iniciativas empresariales en etapa de formación (SENA, 2016).

Parafraseando a Kirzner (1999), en el modelo de crecimiento capitalista, el emprendedor es quien está continuamente alerta y sabe aprovechar oportunidades ya que la competencia en la economía capitalista es un proceso dinámico, que resulta incompatible con la concepción neoclásica del desarrollo como un proceso de progresión armonioso en constante evolución. Niega la posibilidad de alcanzar un equilibrio estático, puesto que serán precisamente los emprendedores quienes a través de su actividad lo modifiquen para establecer nuevas posiciones monopolísticas a través de la introducción de innovaciones (p.57).

El impulso al emprendimiento conlleva el diseño de las metodologías adecuadas para que, en la práctica, las ideas sean plasmadas en modelos de negocios que tengan oportunidad de escalar en el mercado hasta convertirse en empresas con la suficiente capacidad productiva para aportar al crecimiento de la economía desde su sector, y por ende al desarrollo local, regional y nacional.

Las metodologías para el desarrollo de negocios cobran especial relevancia en el panorama del posconflicto por lo cual es indispensable contar con las adecuadas para garantizar que los emprendedores puedan, no solamente traducir sus iniciativas a planes de negocios estructurados y coherentes, 
sino que ello les permita acceder a los recursos que les demanda la puesta en marcha del emprendimiento y avanzar hacia formas empresariales sólidas y perdurables en el tiempo.

Un estudio minucioso de la bibliografía actual relacionada con esta temática evidencia que, actualmente y a pesar de que existen infinidad de propuestas relacionadas con el emprendimiento y los emprendedores, sólo hasta hace poco, con la aparición de los CANVAS - lienzos o tableros - se da un salto significativo que permite superar el paradigma de los planes de negocios que se habían convertido en el "sine qua non" del planeamiento para los nuevos emprendimientos y prácticamente en la única carta de referencia y presentación de los proyectos de empresa para los fondos de inversión y de los inversionistas en general, dando como resultado que la gran mayoría de esas iniciativas fracasaban inexorablemente en el corto plazo.

Sobre los planes de negocio, Claude Ananou (2015) afirma que el plan de negocios ya no es la herramienta principal para enseñar emprendimiento o empezar una empresa; y en esto coincide con la Agencia Para la Creación de Empresas de Francia (APCE, 2011), que ha mencionado que alrededor del $95 \%$ de los planes de negocio no funcionan, esto debido a la complejidad de la predicción del comportamiento futuro del entorno. Por otro lado, existe una parte emotiva al momento de escribir un plan de negocios que hace que el futuro emprendedor tenga una óptica favorable dando una visión positiva de la empresa y en ocasiones poco realista.

El informe de Evaluación del Fondo Emprender del SENA (2013), concluye de igual manera que la "mortandad" de las empresas en Colombia calculada en un $35 \%$ aproximado es muy alta, no obstante estar basadas en planes de negocios, asesoradas y acompañadas por el FE, tanto en el diseño de los mismos como en su posterior implementación.

Surge así la pregunta acerca del futuro de las iniciativas empresariales de quiénes, como beneficiarios del posconflicto, recibirán apoyos económicos para financiar sus ideas de negocios y quiénes contarán con las herramientas 
adecuadas de planeamiento pero sobre todo de identificación de aquellos productos y servicios que, generando valor para sus consumidores y usuarios, los convertirán en ciudadanos prósperos y a sus empresas en organizaciones productivas, sostenibles y escalables en el tiempo. Esto es en últimas lo que se espera de los actores involucrados directa o tangencialmente en las dinámicas de una sociedad que tras años de estar golpeada por los duros embates de la guerra tienen la responsabilidad, desde el desarrollo económico de sus individuos, de sacar adelante sus emprendimientos de estabilización económica, la mayoría en el ámbito rural, para lograr la plena restauración y reincorporación de sus vidas y las de sus familias al constructo general de una Colombia en paz.

Dado lo anterior, la presente investigación ha sido desarrollada y orientada a responder a esta necesidad de adaptar la metodología de los canvas a las exigencias de la economía colombiana en situación de posconflicto, cuyas condiciones hacen que los requerimientos y la importancia de los factores que afectan una empresa naciente tengan necesidades especiales. Se establece así como objetivo principal: identificar el modelado canvas adecuado para los nuevos negocios de posconflicto en Colombia; y como objetivos específicos: 1) conocer el estado del arte de los métodos de prospectiva para el montaje de nuevos negocios; 2 ) identificar las variables que intervienen en el modelado de los nuevos negocios en Colombia en época de posconflicto; y 3) identificar las técnicas de validación para cada variable del metodo identificado.

\section{Contenidos}

Dadas las características del contexto colombiano del posconflicto y teniendo en cuenta que se trata de emprendimientos nuevos, son necesarios elementos de innovación y estrategia, así como de generación y entrega de valor los cuales garantizan el éxito de las iniciativas de los emprendedores. La conjunción correcta de estas variables se logra mediante la aplicación de un adecuado modelo de negocio que es "la forma como la empresa va a lograr ganancias con 
los productos y/o servicios que ofrece, generando una experiencia de compra valiosa para el cliente a través de estrategias innovadoras en la cadena de vaIor" (Weinberger, 2009). En otras palabras, el modelo de negocio, "describe la lógica cómo una empresa crea, entrega y captura valor" (Osterwalder, 2010). Es una herramienta conceptual que, mediante un conjunto de elementos y sus relaciones, permite expresar la lógica mediante la cual una compañía intenta ganar dinero generando y ofreciendo valor a uno o varios segmentos de clientes, la arquitectura de la firma, su red de aliados para crear, mercadear y entregar este valor, y el capital relacional para generar fuentes de ingresos rentables y sostenibles (Osterwalder, 2010).

Una encuesta, a nivel mundial, de la Unidad de Inteligencia de Revista Economist, efectuada a más de 4.000 altos directivos, encontró que la mitad de ellos favorecía nuevos modelos de negocio, como fuente de ventajas competitivas. El mensaje que esos ejecutivos dieron fue claro y contundente: "la forma en que las empresas hacen negocios, será más importante que el producto o servicio que ofrezcan" (Pereira, 2014). Las innovaciones, para mejorar los procesos y productos, requieren fuertes inversiones en tiempo y dinero. Toda innovación requiere de investigación y desarrollo de recursos especializados, nuevas plantas, equipos y unidades de negocios. El futuro rendimiento de esas inversiones es incierto.

Como alternativa o complemento a la creación de productos las empresas le apuestan a la 'innovación en los negocios'. Las investigaciones de la Unidad de Inteligencia del Economist, muestra que, en un mundo altamente interconectado, con recursos financieros escasos, los empresarios y gerentes deben mirar más allá del producto y los procesos productivos. Deben centrarse en innovar el modelo de negocio que actualmente tienen. Un nuevo modelo de negocio puede crear y aprovechar las oportunidades de ingresos y beneficios, contrastando con modelos envejecidos, que llevan a las empresas a la disminución de los ingresos y eventualmente al cierre (Pereira, 2014). 
Amit y Zott definen el modelo de negocios como:

La forma en que la empresa hace negocios con sus clientes, socios y proveedores. Es decir [...] el sistema de actividades específicas que la empresa o sus socios llevan a cabo para satisfacer las necesidades percibidas en el mercado, cómo están relacionadas entre sí esas actividades, y quién las realiza. Los elementos importantes que caracterizan a un sistema de actividades son su contenido, su estructura y su forma de gestión. Un modelo de negocios en particular se explica por las interrelaciones que crea con su entorno: interdependencias relacionadas con la definición de la actividad; interdependencias relacionadas al contenido, a la estructura y a la gestión del modelo de negocios; e interdependencias relacionadas al modelo de ingresos (Zott y Amit, 2009).

Es Osterwalder (2010) quien identifica con mayor precisión los elementos que componen un modelo de negocios. Estos se han establecido como los segmentos de clientes en los cuales se atiende: canales de distribución, propuesta de valor, actividades clave, recursos clave, las relaciones con los clientes, red de asociados, entre otros; todos sobre la base de la estructura de costos del negocio y flujo de ingresos del mismo. De la forma innovadora como se combinen estos elementos se determina un nuevo modelo de negocios.

La aplicación de mayor impacto de la propuesta de Osterwalder es su uso para la innovación de modelos de negocio. En esta metodología se constata que el atributo del poder comunicativo de los nueve bloques, con su visión holística, se combina con la creación del modelo por parte de personas que conocen los diferentes aspectos de un negocio y su entorno competitivo. Todo esto se hace con ejercicios presenciales de generación de ideas, con el uso de las tormentas de ideas y otras herramientas.

Son tres los pasos fundamentales en el diseño de modelos de negocio sugerido por Osterwalder (2010): visualizar el modelo, evaluar e innovar. Posteriormente se lleva a la práctica el diseño, lo que incluye los pasos de planear el proyecto, comunicar e implementar. 
En el modelo canvas se maneja una estructura en donde, por un lado, se ubica el mercado; y por otro, la empresa, entorno, procesos y sus activos, lo cual es perfectamente aplicable a empresas con trayectoria y experiencia, no es muy conveniente a la hora de trabajar con emprendedores y empresas nuevas, porque aún no se sabe con exactitud cuál es la empresa que se está construyendo. Lo anterior explica por qué es necesario considerar otra posibilidad como la que plantea Maurya (2012) para la consolidación de un nuevo emprendimiento: "[...] la metodología CANVAS ha sido desarrollada especialmente para las start ups sin haber profundizado en general en su aplicación en las empresas tradicionales" (p. 65), entendiendo que con éste término se designa a aquellas empresas que están por fuera de internet, es decir, aquellas cuya actividad económica puede ser realizada sin la ayuda de la web.

Es muy preocupante que dichas metodologías tan populares y conocidas en Estados Unidos, particularmente, no se apliquen masivamente en Colombia, en donde las condicones de la economía y del mercado, sobre todo en el marco de las estrategias de generación de empleo para los desmovilizados y víctimas del conflicto, hacen que no solo se requiera de manera urgente el uso de este tipo de herramientas para el emprendimiento, sino que además, esas herramientas sean adaptadas a las particularidades de una economía emergente cuyas características hacen que factores como el riesgo, la competencia o la financiación, adquieran una relevancia especial sobre otros que se consideran en los canvas en general para otro tipo de economías mas desarrolladas.

La forma de llevar al mercado una empresa nueva es completamente diferente a la estrategia de crecimiento o diversificación de una compañía consolidada. Para ello han surgido interesantísimas metodologías como Lean Startup, propuesta por Eric Ries (2011) en su libro El método Lean Startup, que parte del supuesto de que una startup es una organización temporal cuyo objetivo es encontrar un modelo de negocio viable y escalable mediante una serie de experimentos que sirven para aprender en medio de un contexto de incertidumbre. Teniendo claro este supuesto, la metodología Lean Startup propone un ciclo de aprendizaje enfocado en el cliente y no en el 
producto. La idea es desarrollar un MVP (Producto Viable Mínimo), que es un prototipo de nuestro producto con sus características fundamentales, para poder testearlo con clientes reales y maximizar nuestro aprendizaje sobre el negocio, el producto y el mercado. Este ciclo de aprendizaje se compone de tres etapas: "Construir - se desarrolla un MVP tomando como referencia las hipótesis que deseamos poner a prueba- Medir - se establecen las métricas para evaluar nuestro experimento- y Aprender -con la información obtenida podemos mejorar nuestro producto o modelo de negocio-" (Ries, 2011).

Posteriormente Ash Maury (2012) propuso un método donde hibrida ambos mundos, y en el que construye una herramienta para que las empresas nuevas puedan diseñar modelos de negocio: el Lean Canvas, el lienzo de modelos de negocio para empresas nuevas. Lean Canva es una herramienta mucho más apropiada para un nuevo negocio en el que la incertidumbre es la condición predominante (Maurya, 2012)

\section{LIENZO LEAN CANVAS}

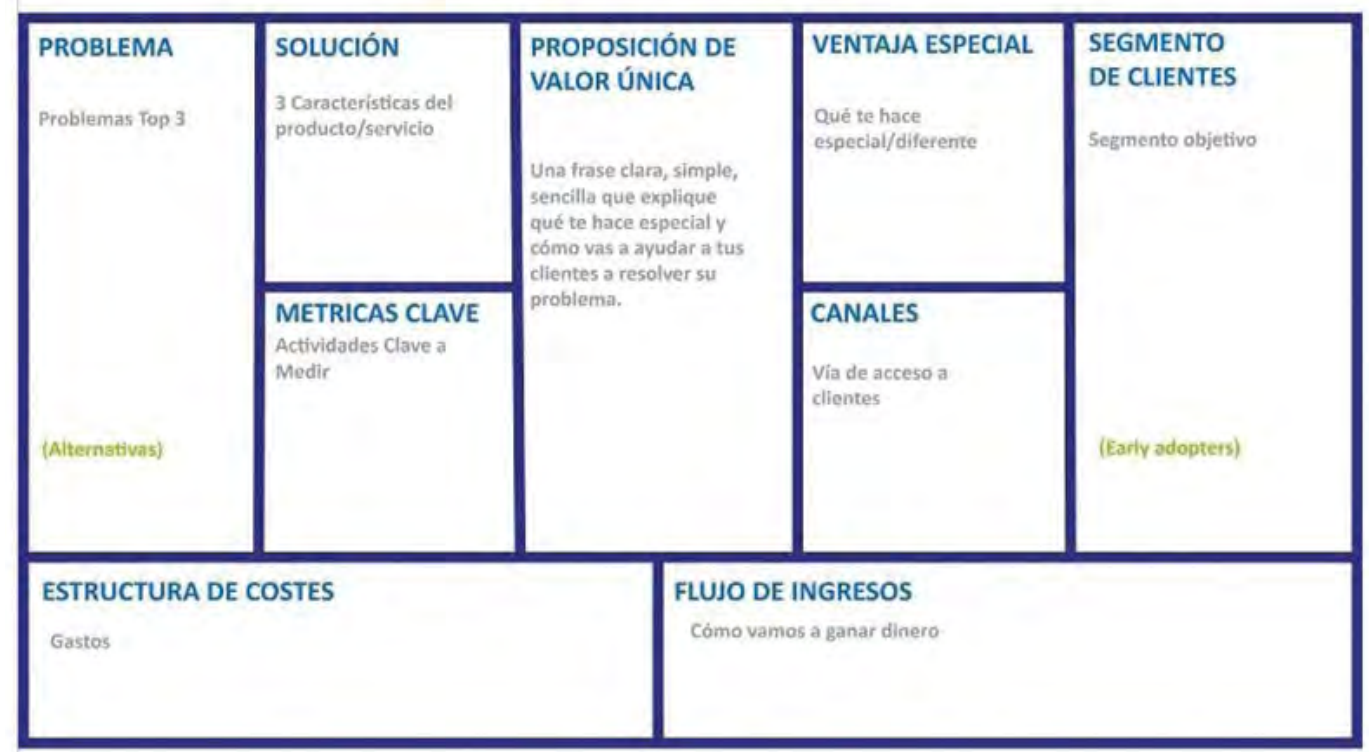

Figura 1. Lean Canvas

Fuente: Lid learning, 2016 
A continuación se describen los cambios mencionados colocando en primer lugar el nombre según el modelo canvas y seguidamente el nuevo nombre del bloque según el Lean Canvas para empresas nuevas:

- Alianzas -> Problema: un aspecto clave en cualquier negocio, sobre todo para una startup, es tener claro el problema o problemas que resuelves, y por eso aparece este bloque.

- Actividades clave -> Solución: de forma similar, y una vez especificado el problema, es conveniente definir las funcionalidades principales del producto que ayudarán a resolver esos problemas

- Recursos clave $\rightarrow$ Métricas clave: para cualquier startup es absolutamente crítico definir desde el principio los tres o cuatro indicadores que va a utilizar para medir el modelo de negocio, elemento clave a la hora de reinventarnos.

- Relaciones -> Ventaja especial/diferencial: este bloque recoge algo que es muy difícil de cuantificar, pero que sin embargo es la clave de cualquier negocio y que suele ser la clave del triunfo frente a la competencia (Megías, 2012).

El cambio más importante y principal que se produce no es tanto de bloques sino de enfoque: en el Lean Canvas, el lado derecho sigue representando el mercado, pero el lado izquierdo representa el producto — unidad de trabajo más real para una empresa nueva-.

La forma de utilizar el Lean Canvas que propone Ash Maurya en su interesante libro Running Lean: Iterate from Plan A to a Plan That Works (2012) se muestra en la figura 2 -los números indican el orden en el que se deben rellenar los bloques-. 


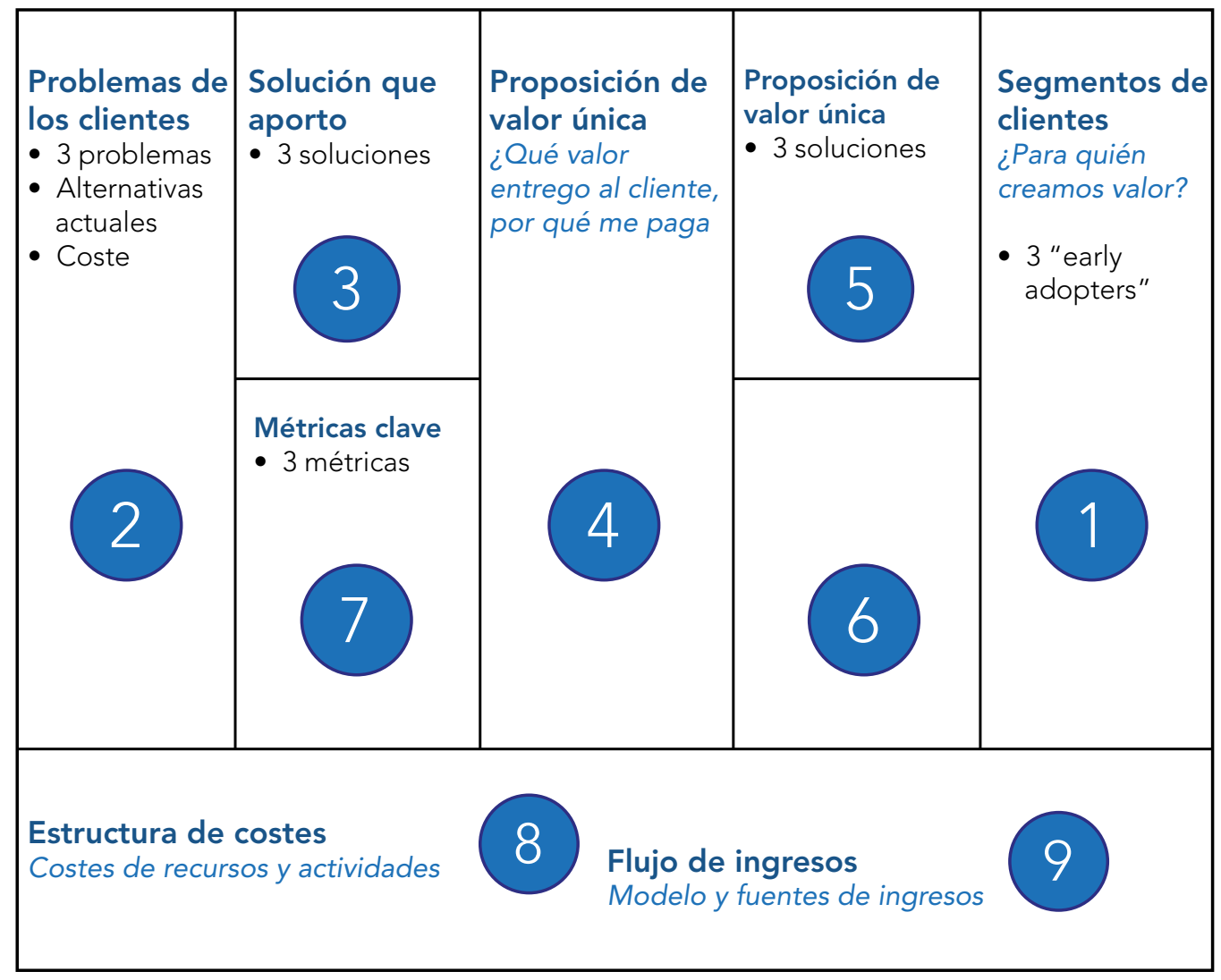

Figura 2. Lean en secuencia

Fuente: (Lid learning, 2016)

A continuación se describen los aspectos del canvas de Osterwalder que se conservan:

- Segmentos de clientes: se identifican y reconocen los segmentos de clientes sobre los cuales trabajar, los usuarios visionarios con los que comenzar a trabajar. Esto es de vital importancia, ya que dirigirse al mercado de masas con usuarios maduros suele ser una mala idea para una startup, al menos de entrada.

- Proposición única de valor: se debe dejar de forma clara, simple, sencilla y describir en una frase concreta qué hace especial a la nueva empresa y su oferta; y cómo esta va a ayudar a los clientes a resolver su problema. 
- Canales: se debe trabajar sobre cómo se va a hacer llegar la solución a los segmentos de clientes con los que se va a trabajar: ¿con una fuerza comercial?, ¿mediante una página web? Es importante entender este camino a los clientes de forma global, es decir, no sólo pensando en la fase de la venta sino en toda la experiencia de cliente.

- Flujos de ingreso: en este punto se debe reflexionar sobre cómo la empresa va a ganar dinero, lo que no sólo incluye pensar en los diversos flujos, sino en el margen, valor del cliente, modelo de recurrencia y demás formas de ingreso.

- Estructura de costes: es el reverso de los ingresos, en la estructura de costes, se deben recoger todos aquellos elementos que cuestan dinero, y que en la práctica indican el gasto aproximado que se tendrá mensualmente y que, por supuesto, al principio deberían ser lo más contenido posible (Megías, 2012).

La diferencia más importante entre éste y el lienzo original de Osterwalder es que el Lean Canvas fue diseñado específicamente para emprendedores, no para consultores, inversores, mentores ni clientes. Se enfoca en entender el problema, como primer requisito, para luego enfocarse en la acción de crear el producto (Serrano y Blazquez, 2015). Por esta razón, ambas herramientas resultan muy útiles para documentar de manera escrita - o impresa - las hipótesis y los aprendizajes claves de un modelo de negocio particular, y como herramienta para poder compartirlos y analizarlos con otras personas y así construir un modelo de negocio exitoso que funcione.

Teniendo en cuenta la particularidad de los emprendimientos que surgirán en las difíciles condiciones del posconflicto, se hace necesario pensar en su diseño en términos de prospectiva,' para lo cual se requiere contar con la metodología

1 Prospectiva es "el proceso que involucra períodos iterativos de reflexión abierta, trabajo en red, consulta y discusión, conduciendo a una visión conjunta y refinada de visiones de futuro y de estrategias comunes de los involucrados ... esto es el descubrimiento de un espacio común para pensar en el futuro y la generación de aproximaciones estratégicas" (Cassigena Harper y Georghiou, 2005) 
adecuada, particularmente para la identificación de los modelos de negocios a implementar como punto de partida. Al respecto Chaquiriand, comenta que "se necesita un modelo de negocio que permita generar dinero, y además, generar diferenciales respecto a nuestra competencia. Pensar en cómo lo vamos a hacer es tan importante como pensar en qué es lo que haremos" (2014).

Por otro lado, si se considera que la mayoría de los emprendimientos falla por problemas en el modelo de negocio, resulta fundamental determinar este modelo de manera que se reduzca al mínimo el riesgo de fracasar en el cumplimiento de los objetivos específicos del negocio proyectado. Pero, ¿cuál es modelo de negocio adecuado para el desarrollo de un emprendimiento en las condiciones del posconflicto?

Los emprendedores aún no saben quién es su cliente o lo que su producto será exactamente. A medida que el mundo se vuelve más incierto, se hace más difícil de predecir el futuro. La planificación y la previsión solo sirven cuando se basan en una larga historia de funcionamiento estable y un ambiente de relativa certidumbre. Los startups no tienen nada de eso. Y por ello es inevitable experimentar, al menor costo posible, para ajustar y volver a planear (Chaquirian, 2014).

\section{Metodología}

Esta es una investigación cuantitativa, descriptiva, correlacional concluyente, dado que se suministra información para la evaluación de datos primarios y secundarios a fin de proveer cursos alternativos de acción. Según la clasificación de Day (2005) es una investigación descriptiva ya que se pueden detallar en forma gráfica los fenómenos y características del análisis de mercado, se determina la asociación de variables y con fundamento en ello se hacen los presupuestos y las predicciones necesarias. 


\section{Resultados}

En cuanto al modelo de negocios para el posconflicto y dado todo lo anterior, se debe tener en cuenta que, para tener éxito en este complicado contexto, es necesario replantear el modelo de negocio con el que se van a desarrollar los productos y servicios, y en general las empresas que los sustentarán en sus mercados objetivo. Dado que la esencia de dicho modelo es la manera en que se proyecta crear, entregar y capturar valor; entonces, de lo que se trata es de la diferenciación en estos aspectos, para lo cual las alternativas deben ser estudiadas a profundidad para determinar cuáles son las más apropiadas y que puedan garantizar al final el éxito de este emprendimiento en términos de rentabilidad económica, de posicionamiento en los mercados local, regional y nacional principalmente y de sostenibilidad en dichos mercados.

Es evidente que los recién llegados inteligentes y exitosos no replican los modelos de negocios existentes, no compiten por canales de distribución repletos ni tampoco van de inmediato detrás de los consumidores mayoritarios. Más bien, atacan los puntos más débiles del enemigo, luego obtienen una ventaja competitiva y más tarde, si les es útil, atacan las fortalezas. Los entrantes exitosos usan tres enfoques básicos en sus ataques indirectos: apalancan sus activos y recursos existentes, reformulan sus cadenas de valor y crean nichos. Estos enfoques podrían parecer simples, pero la magia está en combinarlos. Según Bryce y Dyer, al combinar y unir los enfoques, las empresas pueden desafiar la lógica económica de medio siglo y ganar dinero ingresando en industrias muy rentables (Bryce y Dyer, 2007).

En concordancia con lo anterior y para garantizar el adecuado proceso para la posterior implementación del modelo de negocios diseñado, se hace indispensable practicar un exhaustivo análisis de viabilidad general del proyecto empresarial, cuyo objeto es identificar y delimitar aspectos del mercado, técnicos, institucionales, logísticos y financieros de su gestión. Se busca con esto especificar los planes de inversión y montaje del proyecto, incluyendo necesidades de insumos, estimativos de costos e ingresos, entre otros. 
La evaluación financiera identifica, desde el punto de vista del inversionista, los ingresos y egresos atribuibles a la realización del proyecto y, en consecuencia, la rentabilidad generada por el mismo. Es decir, la evaluación financiera juzga el flujo de fondos del proyecto a la luz del objetivo de generar rentabilidad (Mokate, 2004).

El análisis de viabilidad se hace para mitigar los impactos económicos negativos -ineficiencia de la inversión-, por tanto, debe ser viable desde el punto de vista de la evaluación costo-beneficio que se le debe efectuar. La previsión de la disponibilidad de insumos, de la tecnología a utilizar, de los riesgos, de los mecanismos de comercialización del producto y de la rentabilidad son determinantes para la viabilidad económica del proyecto.

Para llevar a cabo el diseño empresarial del proyecto se deben desarrollar los siguientes estudios específicos:

- Estudio de entorno.

- Estudio de mercado.

- Estudio técnico.

- Estudio legal.

- Estudio administrativo.

- Estudio financiero.

El estudio financiero está integrado por elementos cuantitativos, que permiten decidir y observar la viabilidad económica de un proyecto, en ellos se integra el comportamiento de las operaciones necesarias para que una empresa marche y visualice a su vez el crecimiento de la misma en el tiempo. De ahí, la importancia que al iniciar cualquier idea de proyecto o negocio se contemplen las variables que intervienen en el desarrollo e implementación. Se considera el costo efectivo que conlleva el operar el proyecto en términos financieros e implica el costo de capital de trabajo, adquisiciones de activo fijo, gastos preoperativos y la integración de la información financiera, resumida en los estados financieros proyectados: estados de pérdidas y ganancias. 


\section{Propuesta}

Con fundamento en todo lo expuesto tanto en el apartado de contenido como en el de resultado, se estructura la siguiente propuesta de canvas para los emprendimientos del posconflicto, enmarcados en una política de paz que apunta a lograr la estabilización de miles de personas involucradas en el conflicto colombiano, que ven la posibilidad de reconstruir sus vidas en un ambiente de paz, pero tambien de gran incertidumbre. Sobre todo si se analiza la actividad economica que sustentará sus vidas y las de sus familias, la cual consiste básicamente en la estructuración de pequeñas unidades productivas, que aparte del apoyo económico estatal que recibirán, ${ }^{2}$ requerirán de todo el soporte metológico y técnico en el diseño del modelo de negocio, y el acompañamiento procedimental en la implementación del plan de empresa, que son los requisitos mínimos con los que deben contar para garantizar la estabilización y el escalamiento, que a su vez les permitan posicionarse y mantenerse en las difíciles y riesgosas condiciones de mercado en las que deberán competir.

La propuesta consiste básicamente en acoger la metodología Lean Canvas de Ash Maurya para modelos de negocios tipo start up o empresas nacientes descrita en el contenido. Esta metodología abre las posibilidades para que los emprendimientos que requieran de un diseño de negocio innovador y necesiten en gran medida el éxito de su implementación y desarrollo en el mercado, amplíen sus posibilidades de éxito al basarse en lo aquí expuesto. Especialmente si se abordan los aportes realizados por Maurya al método canvas de Osterwalder, de tal manera que se recomienda basarse en él para que dicho diseño sea una tarea más práctica, eficaz y segura.

En el mismo sentido y dadas las particulares condiciones de los emprendimientos del posconflicto, se propone modificar el Lean Canvas de Maurya agregando un panel denominado riesgo ubicado debajo del que corresponde a métricas,

2 Cada integrante de las FARC-EP en proceso de reincorporación, tendrá derecho por una vez, a un apoyo económico para emprender un proyecto productivo individual o colectivo, por la suma de 8 millones de pesos (Gobierno de Colombia, 2016) 
para que se complemente con éste y permita trabajar y visualizar con la misma metodología prospectiva todo aquello que pudiera amenazar el logro de los objetivos de la nueva empresa. Entendiendo por amenaza todo lo que desde los ambientes externo o interno, pero particularmente desde la situación del actor en su condición de emprendedor de posconflicto, pudiera desestabilizar de alguna manera el normal escalamiento de un emprendimiento bien estructurado en cualquier lugar del país, especialmente en el sector rural de las zonas más afligidas por la guerra.

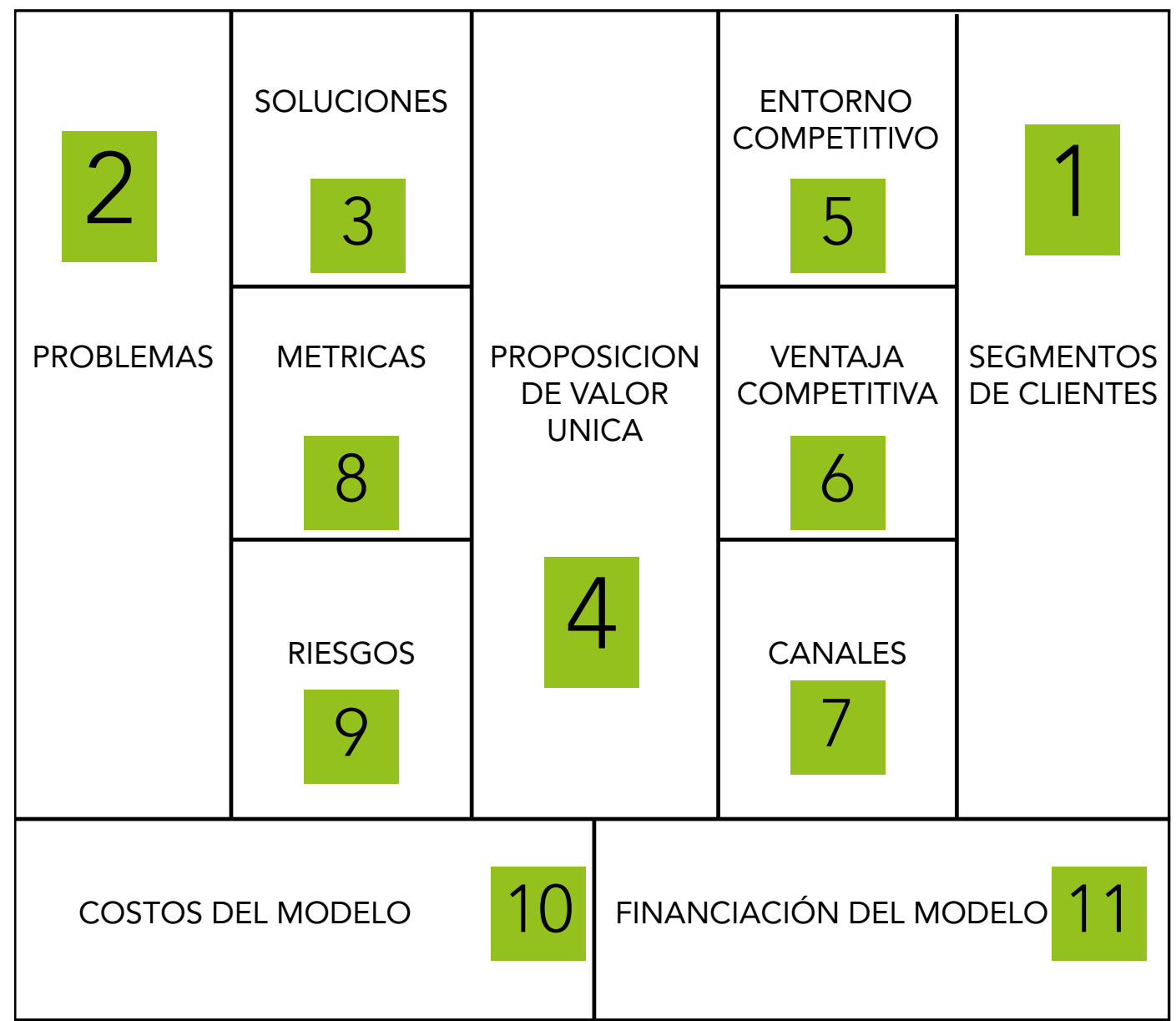

Figura 3. Propuesta

Fuente: elaboración propia 
Se propone una modificación adicional en el panel de ventaja competitiva al cual se le adiciona un nuevo cuadro en la parte superior para trabajar aquí aspectos claves del entorno competitivo donde se iteran los productos mínimos viables y que, a diferencia del panel de riesgo donde se concentra más en las amenazas, relacionadas directamente con el conflicto y sus secuelas, en este nuevo panel se hará énfasis en las condiciones particulares del mercado en el cual el emprendedor buscará su restablecimiento social gracias al escalamiento de su empresa; así, en la mayoría de los casos, también estaría marcado de una u otra forma por las secuelas del conflicto y la persistente economía subterránea ligada al narcotráfico.

En tal sentido será necesario plantear nuevas métricas que, al estilo de la Beta país, permitan calcular no solo el riesgo asociado al emprendedor en sí mismo en su calidad de ex actor del conflicto, sino también del riesgo inherente al ambiente de posconflicto de la zona en la que se desarrollará su emprendimiento.

En este nuevo canvas también se modifica el panel 10, el cual permitirá determinar lo referente al costo específico del modelado que incluye aspectos como el valor de los productos mínimos viables que se iteran, las encuestas y en general todas las estrategias que se emplearán para llegar al producto o servicio de valor único para el segmento escogido.

Se introduce además una modificación en el panel 11, para permitir identificar los recursos que se necesitan para el desarrollo del producto o servicio con propuesta de valor único que posteriormente se lanzará al mercado bajo un esquema de empresa que deberá definirse posteriormente en un plan de negocios en el donde los estudios técnico, de mercado, organizacional y financiero serán fundamentales para garantizar el escalamiento exitoso de la nueva empresa.

De otra parte, se hace necesario orientar nuevas investigaciones a descubrir posibilidades aún inexploradas para los negocios de la región, que tengan en cuenta las particularidades y condicionantes de la misma y permitan identificar la manera más adecuada de intervenir en el mercado con productos nuevos o nuevas formas de comercializarlos. 
También debe considerarse la socialización de los hallazgos aquí expuestos en ambientes académicos de formación para emprendedores y empresarios, dado que estos representan una manera novedosa de llegar con los productos adecuados y ajustados al mercado, y soportados por los análisis como el de producto mínimo viable que dan la posibilidad de ser asertivos en mayor medida en cuanto a las conclusiones que se requieren para satisfacer las demandas de los consumidores y usuarios de los diferentes productos y servicios.

Es pertinente además recomendar el desarrollar de estudios relacionados, orientados a encontrar nuevos métodos para el desarrollo empresarial en las condiciones propias de la cultura y la economía colombiana que puedan ser fácilmente adaptados a las diferentes regiones del país en general.

\section{Conclusiones}

Una vez adelantada la presente investigación y después de revisar la literatura referente al diseño y modelado de negocios como una de sus actividades fundamentales, se puede concluir que la construcción de una metodología, estructurada apropiadamente y lista para la rigurosa organización del proceso de montaje de una nueva empresa (startup), está en pleno desarrollo, y a pesar de los importantes aportes realizados especialmente en la última década alrededor de la temática canvas, la cual ha aportado significativamente al avance de la temática, ésta aún no se puede dar por concluida, especialmente en lo que se refiere al panorama latinoamericano y particularmente el colombiano.

Resulta evidente que para garantizar en mayor medida el éxito en los emprendimientos empresariales de las micro y pequeñas empresas, se hace necesario contar no solo con diseños de negocio apropiados, sino que estos deben ser complementados y soportados por los correspondientes análisis de mercado, técnico, administrativo y financiero que le permitan al emprendedor tener mayor claridad sobre todo el proceso de montaje de su idea de negocio y de su desarrollo en la etapa inicial. 
La construcción exitosa de un modelo de negocio se basa principalmente en la validación de las hipótesis que se identifiquen alrededor de la idea y en las sucesivas iteraciones, que permiten los ajustes necesarios al producto mínimo viable PMV, hasta llevarlo a través de la real puesta a prueba en su nicho de mercado básico (early adopter), y convertirse en un proyecto empresarial viable.

El diseño de negocio aquí presentado, con sus correspondientes estudios de mercado, técnico, administrativo-organizacional y financiero, ofrece un claro panorama para el exitoso montaje de una empresa. Contribuye en gran medida al progreso de los directamente involucrados como el empresario, sus asesores, empleados y sus familias; así como para los beneficiarios indirectos, a saber, los proveedores de materia prima: los agricultores campesinos, productores de los productos y sus derivados, los comercializadores; y especialmente los consumidores del producto que tendrán la posibilidad de tomar un producto que les ofrece ventajas que difícilmente encuentran hoy en día en el mercado.

\section{Referencias}

Bryce, D. y Dyer, J. (2007). Estrategias para penetra mercados bien custodiados. Boston, USA: Harvard Business Review.

Cassigena Harper, J. and Georghiou, L. (2005). The targeted and unforeseen impactsof foresight on ninnovation policy. International journal of forecight and innovation policy: 84-103.

Chaquirian, I. (2014). ¿Por qué fallan los emprendimientos? Sócrates.

Cómbita Mora, G. (2012). Origen y evolución de la teoría del crecimiento impulsado por la demanda real. Scielo-Cuadernos de Economía, 1.

Day, R. (2005). Cómo escribir y publicar. Washington, D.C, USA: The Oryx Press. 
Gobierno de Colombia. (2016). Oficina del Alto Comisionado para la Paz. En línea. Recuperado de: http://www.altocomisionadoparalapaz.gov.co/Paginas/inicio.aspx

Govierno de Colombia. (2016). Urna de Cristal. En línea. Recuperado de: http://www. urnadecristal.gov.co/sites/default/files/acuerdo-final-habana.pdf

Hec montréal mag. (2015). mag.hec.ca. En línea. Disponible en http://mag.hec.ca/wpcontent/uploads/2015/11/Printemps2015.pdf

Kirzner. (1999). Creativity and/or Alertness: A Reconsideration. Review of Austrian Economics, 57.

Lid learning. (2016). Lid learning. En línea. Disponible en http://www. masterempresasdigitales.com/economia-digital/como-buscar-inversores-para-miproyecto-digital-utilizando-la-filosofia-lean/

Maurya, A. (2012). Lean Canvas. Deutso: (s.d.).

Megías, J. (2012). El blog de JAvier Megías. En línea. Disponible en http://javiermegias. com/blog/2012/10/lean-canvas-lienzo-de-modelos-de-negocio-para-startupsemprendedores/

Mokate, K. (2004). Evaluación Financiera de Proyectos de Inversión. Bogotá, Colombia: Alfaomega.

Osterwalder, A. (2010). Business model generation. New Jersey, USA: (s.d.).

Pereira, J. E. (2014). Mercadeo.com. En línea. Disponible en: http://www.mercadeo.com/ blog/2014/02/nuevos-modelos-de-negocios/

Revista Ciencias Estratégicas. (2011). Revista Ciencias Estratégicas. En línea. Disponible en: file:///C:/Users/Juan\%20Carlos/Desktop/Downloads/1090-2793-1-PB.pdf

Ries, E. (2011). El metodo lean startup. Deusto: (s.d.) 
SENA. (2013). Evaluacion Fondo Emprender. En línea. Disponible en: http://www.sena. edu.co/transparencia/gestion-de-planeacion/Documents/Evaluaciones/evalua_ fondo_empren.pdf

SENA. (2016). Fondo Emprender. En línea. Disponible en: http://www.fondoemprender. com/SitePages/QueEsFondoEmprender.aspx

Serrano, M. and Blazquez, P. (2015). Design Thinking: Lidera el presente. Crea el futuro. Madrid, España: Esic.

Silva Colmenares, J. (2007). Crecimiento económico y desarrollo humano. Bogotá, Colombia: (s.d.)

Vargas V, A. (2006). El conflicto interno armado colombiano y sus efectos en la seguridad en la región andina. Ciencia política: 171-172.

Weinberger. (2009). Herramientas para evaluar la viabilidad de un negocio. Lima, Perú: (s.d.)

Zott, C. and Amit, R. (2009). Business Model Design. Elservier.

Zott, C. and Amit, R. (2009). The business model as the engine of network-based strategies. Wharton School Publishing: 28. 